\title{
Construction of cubic Ball surface based on biharmonic partial differentiation equation
}

Azizan Saaban, Ahmad Saleh Kherd, and Noraziah Haji ManSamsul Ariffin Abdul Karim

Citation: AIP Conference Proceedings 1605, 101 (2014); doi: 10.1063/1.4887572

View online: http://dx.doi.org/10.1063/1.4887572

View Table of Contents: http://aip.scitation.org/toc/apc/1605/1

Published by the American Institute of Physics 


\title{
Construction Of Cubic Ball Surface Based On Biharmonic Partial Differentiation Equation
}

\author{
Azizan Saaban $^{a}$, Ahmad Saleh Kherd ${ }^{a}$, Noraziah Haji Man ${ }^{a}$ and \\ Samsul Ariffin Abdul Karim ${ }^{b}$ \\ ${ }^{a}$ Pusat Pengajian Sains Kuantitatif, UUM CAS, Universiti Utara Malaysia, \\ 06010 Sintok, Kedah, Malaysia \\ ${ }^{b}$ Department of Fundamental and Applied Sciences, \\ Faculty of Science and Information Technology, Universiti Teknologi Petronas, \\ Bandar Seri Iskandar, 31750 Tronoh, Perak, Malaysia
}

\begin{abstract}
This paper will discuss a new method of Ball surface generation from prescribed boundaries based on the partial differential operator. In particular, we focus on the construction of a bicubic Said-Ball surface using biharmonic partial differentiation equation. The main result is that the use of biharmonic Said-Ball surface would enable the overall surface to be generated and controlled based on the boundary curves rather than a set of control points. We illustrate the new method by using several graphical examples.
\end{abstract}

Keywords: Said-Ball, Biharmonic, Partial differentiation equation

PACS: 02.60.Ed, 02.30.Jr

\section{INTRODUCTION}

The PDE based on the Laplacian operator and fourth order equation are widely used for surface modeling in CAGD. For example, [1] and [2] constructed the harmonic and biharmonic Bézier surface bounded by the given boundary curves. [3] presents the construction of biharmonic and tetra harmonic Bézier surface with respect to boundary curves and the tangent conditions along them. [4] discussed the methods for designing triangular Bézier PDE surfaces for given different sets of prescribed control points and including the special cases of harmonic and biharmonic surfaces. Currently, [5] presents a new approach to construct surfaces bounded by line of curvature or geodesics based on fourth order PDE. Generally, PDE-based surface modeling has the advantages over the free-form surface modeling based on finding control points because the entire surfaces can be constructed subject to a given set of boundary curves only.

In this paper we will discuss biharmonic surface construction based on Ball surface representation instead of using Bézier representation as stated in the previous researches. We will develop a boundary based intuitive surface design technique for Said-Ball surfaces ([6], [7]) which are one of the basic types of surfaces that are used in Computer Aided Geometric Design (CAGD). The main idea is to find Said-Ball solutions to some natural PDEs which can only be controlled through the boundary control points and those adjacent to them.

The construction of a lower dimension of biharmonic Said-Ball surface of degree 3 by 3 which consists of 16 control points where twelve of them are boundary control points will be discussed. Using the biharmonic PDE, we shall derive a relationship between inner control points with the given boundary control points.

\section{SAID-BALL SURFACE}

Degree $m$ by $n$ Said-Ball patch $S$ is defined as

$$
S(u, v)=\sum_{i=0}^{m} \sum_{j=0}^{n} b_{i j} S_{i}^{3}(u) S_{j}^{3}(v), \quad 0 \leq u, v \leq 1
$$

with $b_{i j}$ are the control points and

Proceedings of the 21st National Symposium on Mathematical Sciences (SKSM21)

AIP Conf. Proc. 1605, 101-105 (2014); doi: 10.1063/1.4887572

(C) 2014 AIP Publishing LLC 978-0-7354-1241-5/ 330.00 


$$
S_{i}^{n}(t)= \begin{cases}\left(\begin{array}{c}
\lfloor n / 2\rfloor+i \\
i
\end{array}\right) t^{i}(1-t)^{\lfloor n / 2\rfloor+1}, & 0 \leq i \leq\lceil n / 2\rceil-1, \\
\left(\begin{array}{l}
n \\
n / 2
\end{array}\right) t^{n / 2}(1-t)^{n / 2}, & i=n / 2, \\
S_{n-i}^{n}(1-t), & \lfloor n / 2\rfloor+1 \leq i \leq n .\end{cases}
$$

where $\lfloor n / 2\rfloor$ and $\lceil n / 2\rceil$ denote the greatest integer less than or equal to $n / 2$ and the least integer greater than or equal to $n / 2$, respectively.

\section{BIHARMONIC EQUATION}

Biharmonic equation for parametric surface $X(u, v)$ is defined as the differential equation obtained by applying the biharmonic operator also known as the bilaplacian, that is the differential operator defined by $\Delta^{4}=\left(\Delta^{2}\right)^{2}$ where

$$
\Delta^{2}=\frac{\partial^{2}}{\partial u^{2}}+\frac{\partial^{2}}{\partial v^{2}}
$$

is the Laplacian and setting to zeros. In general, for rectangular system of coordinates can be written as,

$$
\left(\frac{\partial^{2}}{\partial u^{2}}+\frac{\partial^{2}}{\partial v^{2}}\right)^{2} X(u, v)=0
$$

or

$$
\frac{\partial^{4} X}{\partial u^{4}}+2 \frac{\partial^{4} X}{\partial u^{2} \partial v^{2}}+\frac{\partial^{4} X}{\partial u^{4}}=0
$$

\section{CONSTRUCTION OF BIHARMONIC SAID-BALL SURFACE DEGREE 3 BY 3}

Let $X:[0,1] \times[0,1]$ to be a bicubic Said-Ball surface $(m=n=3)$ as given in (1) represented in matrix form as

$$
X(u, v)=U M V
$$

where

$$
U=\left[\begin{array}{llll}
(1-u)^{2} & 2 u(1-u)^{2} & 2 u^{2}(1-u) & u^{2}
\end{array}\right], V=\left[\begin{array}{lll}
(1-v)^{2} & 2 v(1-v)^{2} & 2 v^{2}(1-v) v^{2}
\end{array}\right]^{T}
$$

and

is the control points matrix.

$$
M=\left[\begin{array}{llll}
b_{00} & b_{01} & b_{02} & b_{03} \\
b_{10} & b_{11} & b_{12} & b_{13} \\
b_{20} & b_{21} & b_{22} & b_{23} \\
b_{30} & b_{31} & b_{32} & b_{33}
\end{array}\right]
$$

Assume that, boundary control points $b_{i 0}, b_{0 j}, b_{i 3}, b_{3 j} i, j=0, \ldots, 3$ are given. We need to determine the function $X$ which satisfy the biharmonic condition as in (4). In order to fullfil these conditions, we will derive the inner points, $b_{11}, b_{12}, b_{21}, b_{22}$ of bicubic Said-ball surface in term of boundary control points. For the bicubic Said-Ball surface, the first and third terms in (4) will be eliminated, leaving only the second term to be considered. Thus, from (4) and (5), we obtain,

where

$$
R M S=0
$$

$$
R=\left[\begin{array}{llll}
2 & 12 u-8 & 4-12 u & 2
\end{array}\right], S=\left[\begin{array}{llll}
2 & 12 v-8 & 4-12 v & 2
\end{array}\right]^{T} .
$$

By expanding (6), we obtain the system of four linear equations in four unknowns and is given as follows: 
where

$$
A Y=B
$$

$$
A=\left[\begin{array}{rrrr}
64 & -32 & -32 & 16 \\
-96 & 48 & 96 & -48 \\
-96 & 96 & 48 & -48 \\
1 & -1 & -1 & 1
\end{array}\right]
$$

is a full rank coefficient matrix, $Y=\left[\begin{array}{llll}b_{11} & b_{12} & b_{21} & b_{22}\end{array}\right]^{T}$ and $B$ is a constant column vector given by $B=\left[\begin{array}{llll}b_{1} & b_{2} & b_{3} & 0\end{array}\right]^{T}$ with

$$
\begin{aligned}
& b_{1}=-\left(4 b_{00}-16 b_{01}+8 b_{02}+4 b_{03}-16 b_{10}-16 b_{13}+8 b_{20}+8 b_{23}+4 b_{30}-16 b_{31}+8 b_{32}+4 b_{33}\right) \\
& b_{2}=-\left(24 b_{10}+24 b_{13}-24 b_{20}-24 b_{23}\right)
\end{aligned}
$$

and

$$
b_{3}=-\left(24 b_{01}-24 b_{02}+24 b_{31}-24 b_{32}\right) \text {. }
$$

Thus, the solution of (7) is

$$
\begin{aligned}
& b_{11}=\left(-b_{00}+2 b_{01}-b_{03}+2 b_{10}+2 b_{13}-b_{30}+2 b_{31}-b_{33}\right) / 4 \\
& b_{12}=\left(-b_{00}+2 b_{02}-b_{03}+2 b_{10}+2 b_{13}-b_{30}+2 b_{32}-b_{33}\right) / 4 \\
& b_{21}=\left(-b_{00}+2 b_{01}-b_{03}+2 b_{20}+2 b_{23}-b_{30}+2 b_{31}-b_{33}\right) / 4 \\
& b_{22}=\left(-b_{00}+2 b_{02}-b_{03}+2 b_{20}+2 b_{23}-b_{30}+2 b_{32}-b_{33}\right) / 4 .
\end{aligned}
$$

The above result shows that the boundaries of a biharmonic Said-Ball surface enables us to fully construct the entire surface.

\section{GRAPHICAL EXAMPLES}

To generate the biharmonic Said-Ball surface, we have chosen three sets of boundary conditions. First set of control points is taken from [3],

$b_{00}=(0,0,0), b_{10}=(3 / 2,0,-3), b_{20}=(3 / 2,0,3), b_{30}=(3,0,0), b_{01}=(0,3 / 2,-3), b_{31}=(3,1,1)$

$b_{02}=(0,3 / 2,3), b_{32}=(3,2,1), b_{03}=(0,3,0), b_{13}=(1,3,-1), b_{23}=(2,3,-1), b_{33}=(3,3,0)$.

Using the above boundary points, the inner control points of biharmonic Said-Ball surface are, $b_{11}=(5 / 4,5 / 4,-3), b_{12}=(5 / 4,7 / 4,0), b_{21}=(7 / 4,5 / 4,0)$ and $\mathrm{b}_{22}=(7 / 4,7 / 4,3)$.

Second example, two boundary curves are defined approximately as semi-circular arcs with radius 1 and 0.5 respectively, while two more boundary curves define on the curves connecting both arcs as given by the following control points.

$$
\begin{aligned}
& b_{00}=(0.5,0,0), b_{10}=(0.2577,0.4,0), b_{20}=(-0.5,0.36,0), b_{30}=(-0.5,0,0), b_{01}=(0.425,0,0.45), \\
& b_{31}=(-0.425,0,0.45), b_{02}=(0.575,0,0.75), b_{32}=(-0.575,0,0.75), b_{03}=(1,0,1), \\
& b_{13}=(0.5153,1,1), b_{23}=(-1,0.72,1), b_{33}=(-1,0,1) .
\end{aligned}
$$


The inner control points are calculated as, $b_{11}=(0.3865,0.7,0.45), b_{12}=(0.3865,0.7,0.75), b_{21}=(-0.75,0.54,0.45)$ and $b_{22}=(-0.75,0.54,0.75)$.

For the last example, we use the following control points,

$$
\begin{aligned}
& b_{00}=(0-0.9271,2.853,0), b_{10}=(-3,0,0), b_{20}=(-2.427,-1.763,0), b_{30}=(0.9271,-2.853,0), \\
& b_{01}=(-0.4364,1.343,0.35), b_{31}=(0.5225,-1.608,0.3), b_{02}=(-0.368,1.133,0.6), \\
& b_{32}=(0.3241,0.9976,0.55), b_{03}=(-0.9271,2.853,1), b_{13}=(-2.853,-0.9271,1), \\
& b_{23}=(-0.9271,-2.853,1), b_{33}=(0.9271,-2.853,1) .
\end{aligned}
$$

and the inner control points are obtained as,

$$
\begin{aligned}
& b_{11}=(-2.8834,-0.59605,0.325), b_{12}=(-2.9485,-0.39585,0.575), \\
& b_{21}=(-1.634,-2.4405,0.325) \text { and } b_{22}=(-1.699,-2.2403,0.575) .
\end{aligned}
$$

Figures 1(a)-3(a) show the control polygons together with its common boundary cuves for Said-Ball of degree 3, while Figures 1(b)- 3(b) show the bicubic Said-Ball surfaces resulting from the biharmonic solution along with the boundary control points. Clearly seen that, the shape of constructing surfaces reproduce the shapes of the boundary curves.

\section{CONCLUSION}

In this work, we have constructed a biharmonic Said-Ball surface of degree 3, where the inner control points depend on boundary control points fullfill the harmonicity conditions of PDE. We show that for a given cubic SaidBall boundary curve, there exists a unique solution of the biharmonic equation with that boundary. The use of biharmonic Said-Ball surface would enable the overall surface to be generated and controlled based on the boundary curves rather than a set of control points.

\section{ACKNOWLEDGMENTS}

The authors are very grateful to the Ministry of Higher Education of Malaysia for providing us with the fundamental research grant scheme (FRGS) S/O number 12380 to enable us to pursue this research. We would also like to extend our gratitude to Universiti Utara Malaysia for supporting first author's attendance at the "Simposium Kebangsaan Sains Matematik ke 21(SKSM21)" in Penang, Malaysia.

\section{REFERENCES}

1. J. Monterde and H. Ugail, International Journal of Computers and Applications 31 (2), 202-2314 (2009).

2. J. Monterde and H. Ugail, Computer Aided Geometric Design 21, 697-715 (2004).

3. P. Centella, J. Monterde, F. Moreno and R. Oset, Computer Aided Geometric Design 26(2), 152-173 (2009).

4. A. Arnal, A. Lluch and J. Monterde, Journal of Computational and Applied Mathematics 235( 5), 1098-1113 (2011).

5. W. X. Huang, H.J.L. Wu and G. J. Wang, Computers and Mathematics with Applications 65, 673-681 (2013).

6. H.B. Said, ACM Transaction on Graphics 8(4), 360-371 (1989).

7. S.M. Hu, G.Z. Wang, and T.G. Jin (1996), Computer Aided Design 28(2), 125-133. 

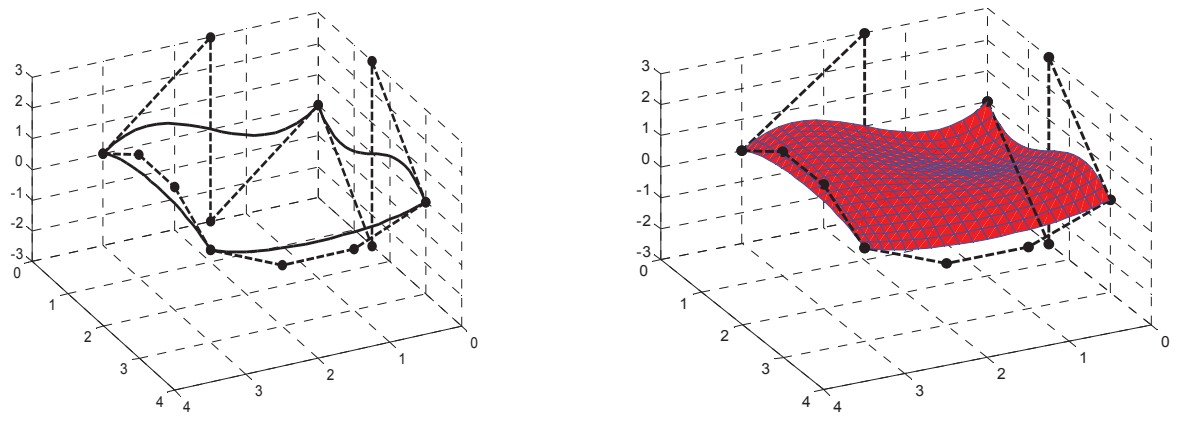

FIGURE 1: a) Control polygon together with its boundary curves (Control points set 1) b) A biharmonic SaidBall surface of degree 3 for the associated boundary curves in (a)
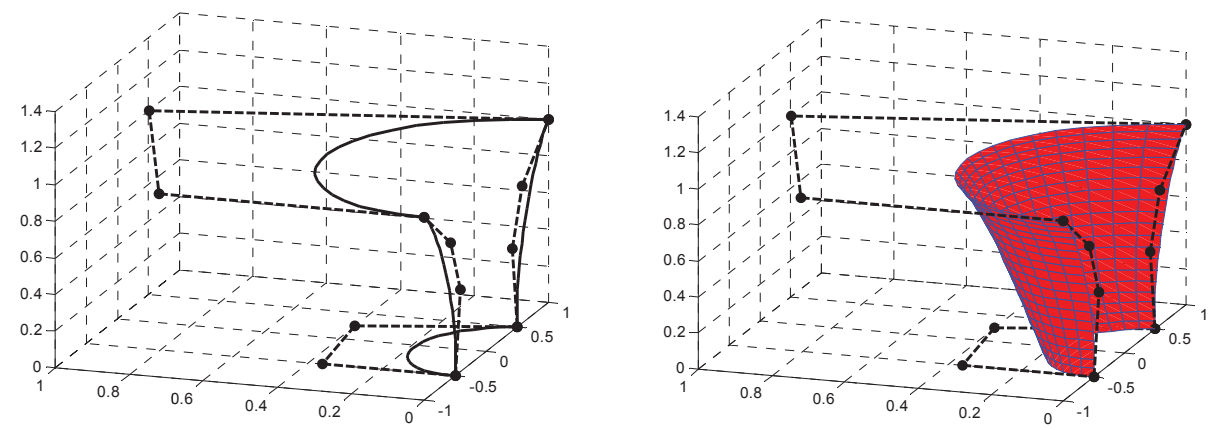

FIGURE 2: a) Control polygon together with its boundary curves (Control points set 2) b) A biharmonic SaidBall surface of degree 3 for the associated boundary curves in (a)
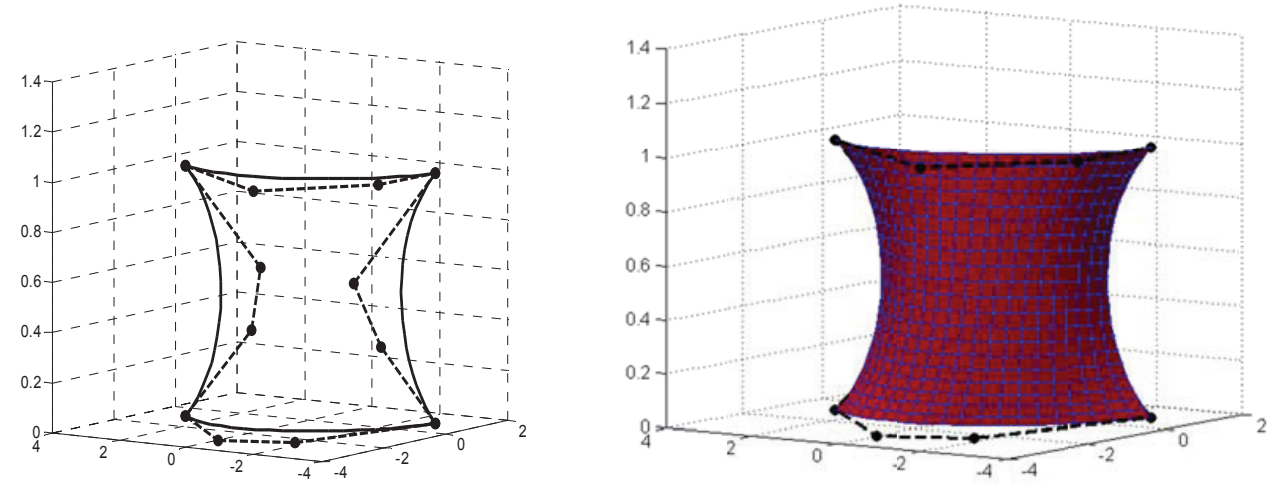

FIGURE 3: a) Control polygon together with its boundary curves (Control points set 3) b) A biharmonic SaidBall surface of degree 3 for the associated boundary curves in (a) 